\title{
A Mean Field Approach for Simulating Wavelength-Division Multiplexed Systems
}

\author{
T. Yu, W. M. Reimer, V. S. Grigoryan, and C. R. Menyuk
}

\begin{abstract}
We simulated many-channel, wavelength-division-multiplexed optical fiber transmission systems by following the full time-domain evolution for only a few channels around a target channel, while treating the rest of the channels as continuous-wave radiation. We show a good agreement between our approach and full numerical simulations. By using our approach, one can greatly reduce the computation time compared with full simulations.
\end{abstract}

Index Terms-Fiber transmission systems, mean field approach.

\section{INTRODUCTION}

B ECAUSE of the dramatic increase in capacity of optical communications using wavelength-division multiplexing (WDM) [1], [2], there has been an increasing interest in the development of efficient simulation tools to model these systems. The split-step method is commonly used [3], [4]; however, this method becomes very computationally time-consuming as the number of channels increases. Roughly speaking, the computational time increases proportional to $N^{2}$, where $N$ is the number of channels. One factor of $N$ is due to the increased bandwidth, and the second factor is due to the smaller propagation step that is required to resolve the dispersive channels as they slide through each other. Here, we discuss a simplified, mean field approach. During the transmission, central channels in a dispersion-managed WDM system are most affected by their nearest neighbors. Channels that are widely separated slide through each other rapidly due to the local dispersion and only affect each other through their average intensity. Thus, it is possible to simulate the behavior of a single channel by retaining the full time-domain evolution for only a few neighboring channels around the target channel. By judiciously choosing the target channels throughout the system bandwidth, it is possible to determine the behavior of the entire system. In principle, this approach reduces the scaling of the computational time from $N^{2}$ to better than $N^{1}$ since the propagation step size becomes independent of the number of channels and the number of target channels that one must keep increases at a rate that is lower than $N$. We note that this approach has been incorporated into commercially available software [5], but it has not previously been described in detail.

Manuscript received August 6, 1999; revised November 8, 1999.

T. Yu, V. S. Grigoryan, and C. R. Menyuk are with the Department of Computer Science and Electrical Engineering, University of Maryland-Baltimore County, Baltimore, MD 21250 USA (e-mail tyu1@umbc.edu).

W. M. Reimer is with the Department of Computer Science and Electrical Engineering, University of Maryland-Baltimore County, Baltimore, MD 21250 USA and also with Ilmenau University, Ilmenau D-98693, Germany.

Publisher Item Identifier S 1041-1135(00)02815-9.
In order to both verify the validity of this approach and to determine the number of neighboring channels that would be required to accurately solve for the behavior of a target channel in a realistic setting, we have carried out a series of simulations for parameters that correspond to undersea systems in which we vary the number of neighboring channels surrounding a target channel. Our dispersion map consisted of one $20-\mathrm{km}$ segment of conventional single-mode fiber with a dispersion of $16 \mathrm{ps} / \mathrm{nm} \cdot \mathrm{km}$ at $1550 \mathrm{~nm}$ and another $160-\mathrm{km}$ segment of dispersion-shifted fiber with a dispersion of $-2 \mathrm{ps} / \mathrm{nm} \cdot \mathrm{km}$ at 1550 $\mathrm{nm}$. The dispersion slope was $0.1 \mathrm{ps} / \mathrm{nm}^{2} \cdot \mathrm{km}$ as is appropriate for fibers with a large effective core area. The total propagation distance is $9900 \mathrm{~km}$. We use a 64-bit pseudorandom signal in each channel with an equal number of marks and spaces, and we verified by testing several different bit sequences that our results did not depend on our choices of these sequences. Each mark is a raised-cosine return-to-zero pulse. The signal is at $10 \mathrm{Gbit} / \mathrm{s}$; so the full-width at half-maximum (FWHM) pulse duration is $50 \mathrm{ps}$. The average power of each channel is assumed to be $0.6 \mathrm{~mW}$, which is reasonable for WDM systems. We model the receiver using a Bessel filter with an 8-GHz bandwidth. These system parameters resemble those of Bergano et al. [2], but the map length is smaller, which we have found yields a better eye diagram.

\section{THEORY}

We may write the equation describing the signal propagation as

$$
i \frac{\partial u}{\partial z}-\frac{\beta^{\prime \prime}}{2} \frac{\partial^{2} u}{\partial t^{2}}+\frac{i}{2} \alpha u+\gamma|u|^{2} u=0
$$

where:

$u \quad$ envelope of the signal wave;

$z$ and $t \quad$ distance and retarded time;

$\beta^{\prime \prime} \quad$ group velocity dispersion;

$\alpha \quad$ loss coefficient;

$\gamma \quad$ Kerr coefficient

For WDM systems with well-separated channels, we may also write

$$
u=\sum_{m} u_{m} \exp \left\{i\left[m \Delta \omega t+\frac{1}{2}(m \Delta \omega)^{2} \beta^{\prime \prime} z\right]\right\}
$$

where $m$ is the channel number and $\Delta \omega$ is the channel spacing. So, for example, in the case of a five-channel system ( $m=$ $-2,-1,0,1,2)$, the equation describing the center channel $u_{0}$ becomes 


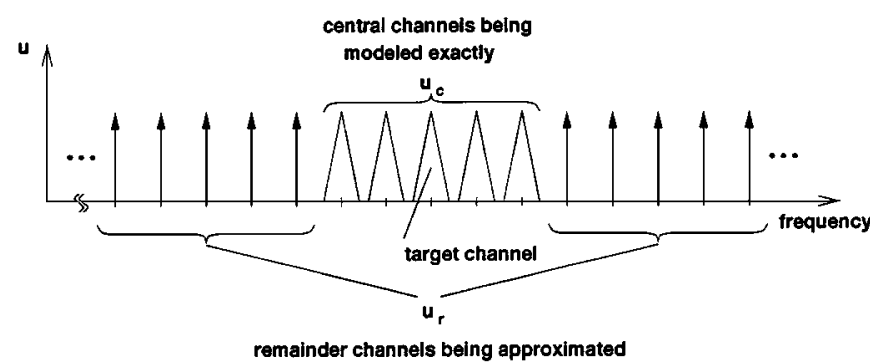

Fig. 1. Schematic illustration of the basic idea behind our mean field approach.

$$
\begin{gathered}
i \frac{\partial u_{0}}{\partial z}-\frac{\beta^{\prime \prime}}{2} \frac{\partial^{2} u_{0}}{\partial t^{2}}+\frac{i}{2} \alpha u_{0}+\gamma\left|u_{0}\right|^{2} u_{0} \\
+2 \gamma\left(\left|u_{-2}\right|^{2}+\left|u_{-1}\right|^{2}+\left|u_{1}\right|^{2}+\left|u_{2}\right|^{2}\right) u_{0} \\
+\gamma\left(u_{1}{ }^{2} u_{2}{ }^{*}+u_{-1}{ }^{2} u_{-2}{ }^{*}\right) e^{i \delta k_{1} z} \\
+2 \gamma\left(u_{-1} u_{1} u_{0}{ }^{*} e^{i \delta k_{2} z}+u_{-2} u_{2} u_{0}{ }^{*} e^{i \delta k_{4} z}\right) \\
+2 \gamma\left(u_{-1} u_{2} u_{1}{ }^{*}+u_{-2} u_{1} u_{-1}{ }^{*}\right) e^{i \delta k_{3} z}=0
\end{gathered}
$$

where $\delta k_{1}=-(\Delta \omega)^{2} \beta^{\prime \prime}, \delta k_{2}=(\Delta \omega)^{2} \beta^{\prime \prime}, \delta k_{3}=2(\Delta \omega)^{2} \beta^{\prime \prime}$, and $\delta k_{4}=4(\Delta \omega)^{2} \beta^{\prime \prime}$. One can see that the four-wave mixing (FWM) terms oscillate rapidly as a function of distance when $\beta^{\prime \prime}$ and the channel separation becomes large. For a three-channel system, there is only one term that contributes to four-wave mixing, corresponding to the term $2 \gamma u_{-1} u_{1} u_{0}{ }^{*} e^{i \delta k_{2} z}$ in (3). For a five-channel system, there are already six terms, and, as the number of channels increases, there is a rapid proliferation of terms. Thus, this decomposition is not useful unless the FWM terms can be ignored. We will show in Section III that the FWM interaction between channels can be ignored for the parameters that we are considering when the channel spacing becomes larger than approximately $1.5 \mathrm{~nm}$. This spacing is large relative to the actual channel spacing in realistic systems, which is 1.0 $\mathrm{nm}$ or less [2]. However, this observation suggests the following procedure: We write

$$
u=u_{c}+u_{r}
$$

where $u_{c}$ contains all the channels within $1.5 \mathrm{~nm}$ of the target channel and $u_{r}$ contains the rest of the channels. We completely follow the temporal evolution of $u_{c}$, while treating $u_{r}$ as continuous-wave radiation, which is equivalent to ignoring the FWM interactions between $u_{c}$ and $u_{r}$, as shown schematically in Fig. 1. Equation (1) is thus replaced by

$$
i \frac{\partial u_{c}}{\partial z}-\frac{\beta^{\prime \prime}}{2} \frac{\partial^{2} u_{c}}{\partial t^{2}}+\frac{i}{2} \alpha u_{c}+\gamma\left|u_{c}\right|^{2} u_{c}+2 \gamma\left|u_{r}\right|^{2} u_{c}=0 .
$$

This approach is not expected to yield an accurate solution for the channels at the edge of the central region included in $u_{c}$ but should yield an accurate solution for the target channel, as we will explicitly verify using simulations in Section III.

A consequence of ignoring FWM contributions between the channels in $u_{r}$ and the channels in $u_{c}$ is that there is no energy transfer between the two sets of channels. Thus, $\left|u_{r}\right|^{2}$ is simply a constant number, independent of $z$, in (5), and it leads to a constant phase rotation of all the channels in $u_{c}$. For point-topoint systems of the sort that we are considering in this paper,

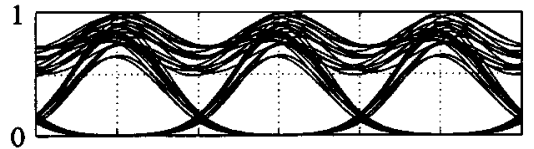

(a)

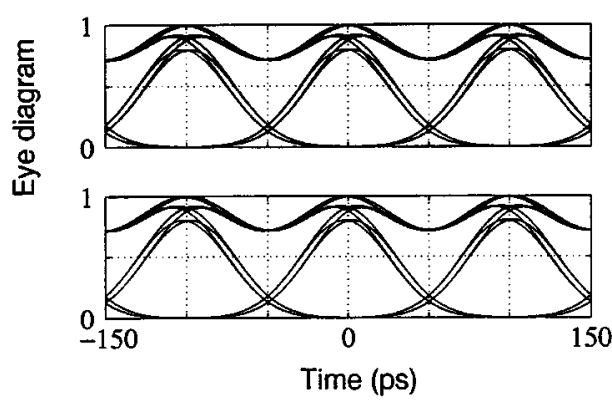

(b)

(c)

Fig. 2. Eye diagrams of the output signal from channel 1 in the two channel system when the channel separation is: (a) 0.5 , (b) 1.5 , and (c) $2.5 \mathrm{~nm}$. The output is shown normalized with respect to the maximum value.

this constant phase rotation has no effect on the received signal for the channels in $u_{c}$ and can be ignored. However, when this technique is extended to networks in which different channels evolve over different distances, it may not be possible to ignore this phase rotation.

\section{Simulation Results}

We begin our numerical investigations by studying the interaction between two channels as a function of the wavelength separation. When the separation is only $0.5 \mathrm{~nm}$, there is a strong FWM interaction between the channels as is evident in the strong pattern dependence of the output signal in Fig. 2(a). By contrast, when the wavelength separation is $1.5 \mathrm{~nm}$, the only pattern dependence that is visible is what is expected for a single channel, as shown in Fig. 2(b). We have verified that when the second channel is replaced by continuous-wave radiation, there is no change in the eye diagram. When the wavelength difference increases further, there is also no change in the eye diagram, as shown in Fig. 2(c). When the wavelength separation is $1.5 \mathrm{~nm}$, we have verified that the power in the FWM products is $30-40 \mathrm{~dB}$ below the signal power.

We now directly investigate the convergence of a WDM system as we increase the number of channels, using the central channel as the target channel. We study cases with three, five, seven, nine, and 11 channels with a spacing of $0.8 \mathrm{~nm}$. Because channels treated in the mean-field approximation only lead to an unimportant phase change in the central channels, as discussed in the previous section, the three-channel simulation is equivalent to an 11-channel simulation in which the eight outlying channels are treated in the mean-field approximation. The five-channel simulation is equivalent to an 11-channel simulation in which the six outlying channels are treated in the mean-field approximation, and so on. The eye diagrams for these five cases, neglecting amplifier spontaneous emission (ASE) noise, are shown in Fig. 3. Normalizing the means and the standard deviations of the marks, measured at the central point of the time window, to the mean-value for the three-channel simulation, we find that the means for the three-, five-, seven-, nine-, and 11-channel simulations are 1.00, 0.963, $0.970,0.984$, and 0.975 and the standard deviations are 0.173 , 


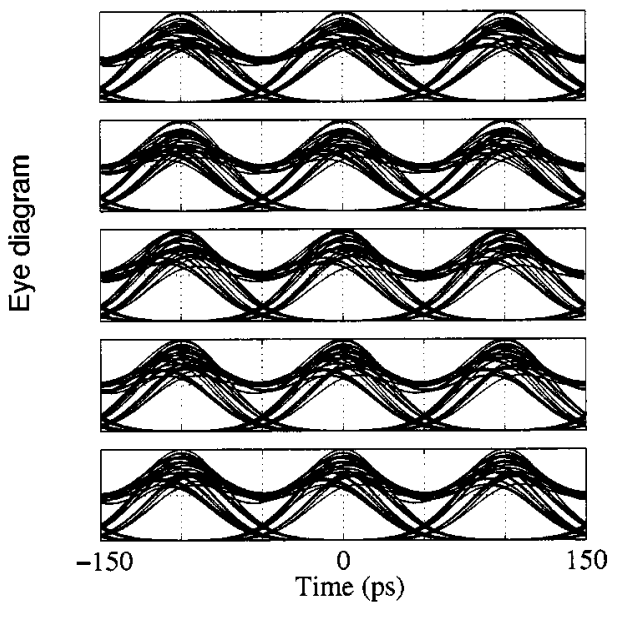

Fig. 3. Eye diagrams of the output signal from the central channel in a WDM system with: (a) three channels, (b) five channels, (c) seven channels, (d) nine channels, and (e) 11 channels. The output is shown normalized with respect to the maximum values.

$0.158,0.157,0.159$, and 0.159 . For five or more channels, these numbers are nearly the same. With three channels, the farthest out channels are only $0.8 \mathrm{~nm}$ from the target channel, which is much less than the requirement of $1.5 \mathrm{~nm}$ predicted by our two-channel simulations, while with five or more channels, the farthest out channel is $1.6 \mathrm{~nm}$ or more. Consequently, the results are consistent with what is expected from the two-channel simulations.

Finally, we add ASE noise to the system and compare full simulations to the mean field approach. The eye diagrams are shown in Fig. 4. In this case, the means are 1.00, 0.99, 1.04, 1.03 , and 1.01, and the standard deviations are $0.272,0.216$, $0.248,0.261$, and 0.254 , respectively. In this case, we need seven channels before the behavior really converges, indicating that one must go somewhat further than what is predicted by the two-channel simulations to obtain reliable results in the presence of ASE noise for reasons that are unclear at present. We have investigated different configuration with target channels at different central wavenumbers, and we have obtained nearly identical results.

\section{CONCLUSION}

In this letter, we have described a mean-field approach that allows us to greatly reduce the computational time that is needed for multichannel WDM simulations by only following the detailed time evolution for a limited number of channels surrounding a target channel and treating the others as continuous-wave radiation. In our approach, we identify the minimum wavelength separation from the target channel that is required for four-wave-mixing contributions to become small

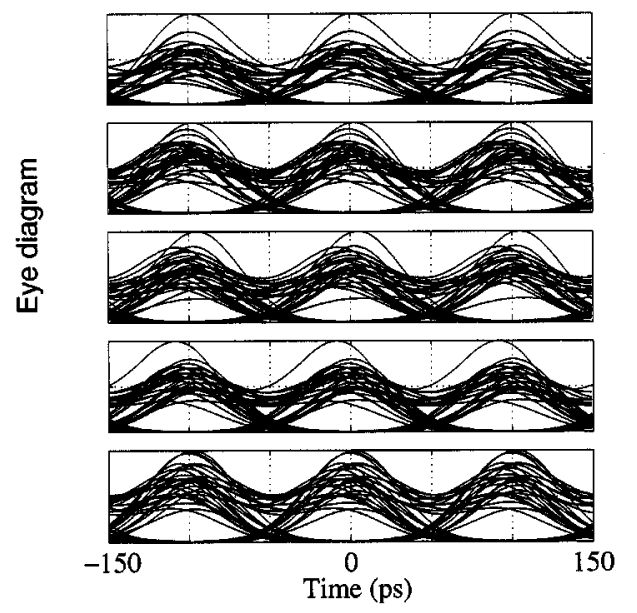

(a)

(b)

Fig. 4. Eye diagrams of the output signal from the central channel in a WDM system with: (a) three channels, (b) five channels, (c) seven channels, (d) nine channels, and (e) 11 channels. ASE noise is present and the output is shown normalized with respect to the maximum values.

by doing two-channel simulations. For point-to-point systems, channels that we treated in the mean-field approximation only lead to an unimportant phase rotation in the central channels and can be ignored. We validated this approach for parameters that are typical for undersea systems. Other simulations that we have made indicate that this approach is also valid for terrestrial systems, although the number of channels that must be kept differs depending on the systems parameters. Using this approach we can significantly reduce the computational time compared to full simulations. The gain is proportional to $N^{2} / N_{\mathrm{mfa}}^{2} N_{\text {target }}$, where $N$ is the total number of WDM channels, $N_{\text {mfa }}$ is the number of channels used in the central region around the target channel, and $N_{\text {target }}$ is the number of target channels that is needed to fully explore the spectra of the system.

\section{REFERENCES}

[1] C. A. Brackett, "Dense wavelength division multiplexing networks: Principles and applications," IEEE J. Select. Areas Commun., vol. 8, pp. 948-964, Aug. 1990.

[2] N. S. Bergano, C. R. Davidson, M. Ma, A. Pilipetskii, S. G. Evangelides, H. D. Kidorf, J. M. Darcie, E. Golovchenko, K. Rottwitt, P. C. Corbett, R. Menges, M. A. Mills, B. Pedersen, D. Peckham, A. A. Abramov, and A. M. Vengsarkar, " $320 \mathrm{~Gb} / \mathrm{s}$ WDM transmission $(64 \times 5 \mathrm{~Gb} / \mathrm{s})$ over 7,200 $\mathrm{km}$ using large mode fiber spans and chirped return-to-zero signals," in Proc. OFC'98, Feb. 1998, PD12.

[3] G. P. Agrawal, Nonlinear Fiber Optics. San Diego, CA: Academic, 1995, p. 242.

[4] D. Marcuse, "Derivation of analytical expressions for the bit-error probability in lightwave systems with optical amplifiers," J. Lightwave Technol., vol. 8, pp. 1816-1823, Dec. 1990.

[5] Photonic Transmission Design Suite: Photonic Modules Reference Manual, Virtual Photonics Inc., Berlin, Germany, 1998. 